\section{The association of the JNK scaffold protein, WDR62, with the mixed lineage kinase 3 , MLK3}

\section{Miriam Hadad, ${ }^{1}$ Sharon Aviram, ${ }^{1}$ \\ Ilona Darlyuk-Saadon, 1 \\ Ksenya Cohen-Katsenelson, ${ }^{1}$}

Alan J. Whitmarsh,2 Ami Aronheim 1

1Department of Cell Biology and Cancer Science, The B. Rappaport Faculty of Medicine, Technion-Israel Institute of Technology, Haifa, Israel; 2Faculty of Life Sciences, University of Manchester, Michael Smith Building, Manchester, UK

\section{Abstract}

Mitogen-activated protein kinases (MAPKs) form a kinase tier module in which MAPK, MAP2K and MAP3K are held by scaffold proteins. The scaffold proteins serve as a protein platform for selective and spatial kinase activation. The precise mechanism by which the scaffold proteins function has not yet been fully explained. WD40-repeat protein 62 , WDR62 is a novel scaffold protein of the c-Jun N-terminal kinase (JNK) pathway. WDR62 is a 1523 a.a. long protein with no significant sequence homology to a known gene. Previously WDR62 was shown to associate with JNK and MKK4/7 in a modular fashion. Here, we show that WDR62 is able to associate with multiple members of the MAP3K of the mixed lineage kinase family and we map WDR62-MLK3 interacting domains. We identify two separable interacting domains within WDR62 and MLK3 proteins that can cross associate. MLK3 association with WDR62 is independent of JNK and MKK4/7 domains and activities. CDC42 activation disrupts WDR62-MLK3 association independent of MLK3 kinase activity.

\section{Introduction}

Mitogen-activated protein kinases (MAPKs) regulate a variety of cellular processes in response to extracellular signals. MAPKs are activated through a protein kinase cascade in which a MAP3K activates a MAP2K that in turn activates a MAPK.1,2 Three main modules exist in mammals: the extracellular regulated kinases (ERK), stress activated protein kinases (SAPK, also known as c-Jun N-terminal kinase, JNK) and p38.3,4 Although all MAPKs recognize an identical phosphoacceptor site, i.e., a serine/threonine residue followed by a proline residue, MAPKs phosphorylate their substrates with great selectivity. In many instances, the MAPK modules have been shown to regulate distinctly different cellular responses in a cell type- or signal-specific manner.5,6 Multiple mechanisms contribute to signal specificity including docking domains, conformational changes, positive and negative feedback loops, and cross-pathway regulatory relay protein tethering..$^{7-9}$ During the last decade, the importance of scaffold proteins in providing signal specificity and fidelity has emerged.10,11 Scaffold proteins play a major role in the efficiency of signal transmission mediating the temporal and spatial signals to their appropriate cellular target, in order to obtain the proper cellular response. 12 However, the precise mechanism of action by which scaffold proteins function remains obscure. ${ }^{13}$ Scaffold proteins act as multi-domain interacting surfaces that serve as a meeting platform for kinases and substrates to orchestrate specific transmission of signaling. 11,13 The JNKs are critical mediators of growth factors, cytokines and stress signals. ${ }^{6,10} \mathrm{JNK}$ is connected to multiple signaling pathways by feed-forward and negative feedback loops allowing flexibility and threshold signal integration within the JNK network conferring a wide range of biological responses. ${ }^{14} \mathrm{JNK}$ is activated by the MAP2Ks, MKK4 and MKK7, via phosphorylation of a TXY motif. 15 These MAP2Ks are activated by multiple MAP3Ks, in particular members of the MEKK, MLK, TAK1 and ASK1 family. 16 Numerous scaffold proteins coordinate the JNK signaling pathway including the JIP (14), ${ }^{17}$ IkB kinase complex-associated protein (IKAP) ${ }^{18}$ and Plenty of SH3 (POSH) proteins. ${ }^{19}$ Previously, we isolated WDR62 as a novel JNK interacting protein using the Ras recruitment system. ${ }^{20,21}$ WDR62 associates with both JNK and MKK4/7 and therefore is considered a bona fide JNK scaffold protein.22 We mapped the JNK and MKK4/7 binding domains to a.a. 1294-1301 and 1212-1284 respectively. ${ }^{22}$ WDR62 is cytoplasmic during interphase and localizes to the spindle pole in mitosis. ${ }^{23}$ Interestingly, WDR62 was found to be mutated in microcephaly patients. ${ }^{24-26}$ Mutations within the gene impair either WDR62 expression or WDR62 centrosome localization. ${ }^{4}$ WDR62 is required for normal cell division of neuroprogenitor cells. WDR62 deficiency results in premature differentiation into immature neurons. ${ }^{27}$ In addition, mutations resulting in truncation of the WDR62 C-terminus were found to impair WDR62 dimerization and the WDR62 monomer fails to recruit JNK to the complex. ${ }^{28}$ The WD40 repeat of WDR62 is responsible for microtubule association. WDR62 specifically recruits JNK1 to the spindle pole which is required for spindle regulation and mitotic progression. ${ }^{29}$ Following arsenite treatment, WDR62 is localized to
Correspondence: Ami Aronheim Department of Molecular Genetics, The B. Rappaport Faculty of Medicine, Technion-Israel Institute of Technology, 7 Efron St. Bat-Galim, Haifa Israel 31096 .

Tel.: (972).4.8295454 - Fax: (972).4-8295225.

E-mail: aronheim@tx.technion.ac.il

Key words: c-Jun N-terminal kinase; MLK3; Scaffold; WDR62.

Acknowledgments: we wish to thank Prof. Kathleen A. Gallo from the Michigan State University for MLK3 deletion constructs, Prof. Rosamaria Ruggieri from the Feinstein Institute for Medical Research, Manhasset, New York for Flag-ZAK expression plasmid and Prof. Anning Lin from the Ben May Institute Chicago for JNKK2-JNK1 chimera.

Funding: this work was supported by: the Israel Science Foundation grant \# 573/11 to A.A. and Welcome Trust 088232/Z/09/Z to A.J.W.

Contributions: $\mathrm{MH}$, data acquisition, data analysis, figures preparation, manuscript preparation; SA, study design, data analysis and manuscript preparation; ID-S, study design and data analysis; KC-K, study design and data analysis; AJW, study design, reagents, data analysis; AA, study design, data analysis, manuscript preparation.

Conflict of interest: the authors declare no conflict of interest.

Received for publication: 9 June 2015. Revision received: 21 September 2015 Accepted for publication: 17 October 2015.

This work is licensed under a Creative Commons Attribution NonCommercial 3.0 License (CC BYNC 3.0).

(C) Copyright M. Hadad et al., 2015

Licensee PAGEPress, Italy

MAP Kinase 2015; 4:5307

doi:10.4081/mk.2015.5307

stress granules and is suggested to mediate mRNA homeostasis following stress. ${ }^{21}$ Although there is much evidence for the role of WDR62 as a JNK scaffold protein in normal cell function and following stress stimuli, the interaction of WDR62 with MAP3K protein has not been characterized. Here, we characterize WDR62 association with the MAP3K, MLK3, a member of the mixed lineage family (MLK). The members of the MLK family are serine/threonine kinases that regulate signaling pathways through activation of the JNKs. ${ }^{16}$ We map and characterize the WDR62-MLK3 association domains and describe the possible role of WDR62-MLK3 association in maintaining MLK3 in the unphosphorylated inactive form. 


\section{Materials and Methods}

\section{Antibodies}

The following antibodies were used: monoclonal anti-HA (clone 12CA5, Abcam), monoclonal anti-Myc (clone 9E10, Abcam), anti-AU5 (ab130113, Abcam), anti GAPDH polyclonal (sc-25778), anti-Flag (M2) monoclonal (Sigma-Aldrich, F1804), anti-pJNK (SigmaAldrich, J4644), and anti-pMLK3 277T/281S (LSBio, LS-C342166). Polyclonal anti-GST antibody was made in our laboratory. ${ }^{21}$ Secondary antibodies were obtained from Sigma-Aldrich (anti-rabbit A0545, anti-mouse A0168) and Jackson ImmunoResearch Laboratories (antimouse light chain 115-035-174).

\section{Plasmids}

The mammalian expression plasmid pCAN was used to express all Myc-tagged human WDR62.28 All deletion constructs were designed according to the amino acid position within the human WDR62. The various fragments were amplified by PCR using appropriate oligonucleotides to allow subsequent cloning by restriction enzymes. Oligonucleotide sequences can be provided upon request. All plasmids were verified by DNA sequencing. The expected size of all fusion protein used in this study are shown in Supplemental Table 1. WDR62 fragments fused to GST (glutathione S-transferase) were cloned into the mammalian expression plasmid pcDNA3-AIRAP-GST-V3, 30 by replacing the AIRAP fragment with WDR62 fragments.

The mammalian expression plasmid pcDNA 3XHA was used to express HA-MLK3 and MLK2.

Kinase dead MLK3 mutant, HA-MLK-K144A, was generated using the QuickChange ${ }^{\circledR}$ sitedirected mutagenesis kit (Stratagene, 200516) according to the manufacturer's instructions. Mutations were verified by DNA sequencing.

HA-tagged human JNK2, DLK, ZAK-, and AU5 tagged CDC42-QL were expressed using the pCEFL mammalian expression plasmid. pcDNA-Flag-MLK3 was constructed by PCR.

PCGN-HA-MLK3-1-114, 115-364, 400-591, 592-847 were kindly provided by Prof. Kathlleen A. Gallo from Michigan State University.

PCMV-Flag-ZAK- was kindly provided by Prof. Rosamaria Ruggieri. ${ }^{31}$

PEBG-DLK-1-407 and 405-888 were described. ${ }^{32}$ pCDNA-HA-JNKK2-JNK1 and pCDNA-HA-JNKK2-JNK1-KM were kindly provided by Prof. Anning Lin from Ben-May Institute, Chicago. 33

\section{Cell culture and transient transfection HEK-293T cells were maintained in}

Dulbecco's modified Eagle's medium (DMEM) containing $10 \%(\mathrm{v} / \mathrm{v})$ fetal bovine serum (FBS), 100 i.u./mL penicillin and $0.1 \mu \mathrm{g} / \mathrm{mL}$ streptomycin and grown at $37^{\circ} \mathrm{C}$ and $5 \% \mathrm{CO}_{2}$. HEK-293T cells were transfected with the appropriate expression plasmids using the calcium phosphate method. 34 The total amount of plasmid DNA was adjusted to $12 \mu \mathrm{g}$ in a total volume of $1 \mathrm{~mL}$. Cell culture medium was replaced with fresh medium 4-5 h post-transfection and cells were harvested 24 h posttransfection.

\section{Co-immuno-precipitation}

Transfected cells were lysed in whole cell extract (WCE) buffer [25 mM HEPES pH 7.7, $0.3 \mathrm{M} \mathrm{NaCl}, 1.5 \mathrm{mM} \mathrm{MgCl}_{2}, 0.2 \mathrm{mM}$ EDTA, $0.1 \%$ Triton X-100, $0.5 \mathrm{mM}$ DTT, $20 \mathrm{mM} \beta$-glycerophosphate, $0.1 \mathrm{mM} \mathrm{Na} \mathrm{VO}_{4}, 100 \mu \mathrm{g} / \mathrm{mL}$ PMSF, Protease inhibitor cocktail 1:100 (Sigma Aldrich, P8340)].

Protein extract (400-700 $\mu \mathrm{g}$ in WCE, buffer) was pre-cleared with non-relevant antibodies bound to protein-A sepharose beads. The lysate was then incubated overnight at $4^{\circ} \mathrm{C}$ with the relevant antibodies. Protein A-Sepharose beads (Sigma-Aldrich, P3391) were then added to the lysate and incubated for $1 \mathrm{~h}$. Samples were extensively washed four times with WCE buffer. The precipitated proteins were eluted by SDS/PAGE sample buffer and then processed for Western blot analysis. Total lysate shown represent $10 \%$ of the lysate used for immuno-precipitation.

\section{GST pull-down assay}

Glutathione-agarose beads (Sigma-Aldrich, G4510) were pre-blocked with 5\% BSA (w/v). The beads were then incubated with 350-700 $\mu \mathrm{g}$ of the protein extract (in WCE buffer) overnight at $4^{\circ} \mathrm{C}$. Following four washes with WCE buffer, the precipitated proteins were eluted using freshly made glutathione elution buffer containing $50 \mathrm{mM}$ Tris-HCl, $\mathrm{pH}$ 8.0, 20 mM L-glutathione (Sigma-Aldrich, G4251), 1 $\mathrm{mM}$ DTT and $1 \mathrm{mM}$ PMSF. Samples were processed for Western blot analysis. Total lysate shown represent $10 \%$ of the lysate used for GST-pulldown.

\section{Western blot}

Cell lysates or precipitated protein complexes were separated by $10 \%$ SDS-PAGE, and electro-transferred to nitrocellulose membranes. Membranes were blocked in 5\% dry milk in PBS or TBST for $0.5 \mathrm{~h}$, and then incubated with primary antibodies for $1 \mathrm{~h}$ at room temperature or overnight at $4^{\circ} \mathrm{C}$. After 3 washes, the membranes were incubated with HRP-conjugated secondary antibodies for $1 \mathrm{~h}$ at room temperature, followed by another 3 washes. Protein bands were detected by enhanced chemiluminescence.

\section{Results}

\section{WDR62 association with MLK family}

The MLK family consists of nine members clustered into three main subgroups based on similarity within their catalytic domain. This includes MLKs, dual-leucine-zipper bearing kinases (DLKs) and Zipper sterile--motif kinases (ZAKs). ${ }^{16}$ To identify interactions between WDR62 and MLK family members, HEK-293 cells were co-transfected with plasmids encoding full length Myc-tagged WDR62 and HA-tagged MLK2, MLK3, ZAK or DLK1. Cell lysates were prepared $24 \mathrm{~h}$ following transfection and used for co-immuno-precipitation (co-IP) assays. The expression levels of all transfected proteins were variable but detectable, as shown by Western blot analysis of total cell lysates (Figure 1, middle and lower panels). We observed strong effect on the expression levels of co-transfected proteins. Interestingly, all the tested HA-tagged MLK family members co-precipitated efficiently with Myc-WDR62 (Figure 1, top panel).

\section{Mapping WDR62 association domains with MLK3}

As a paradigm for interaction between WDR62 and MLK family members, we decided to further characterize the WDR62-MLK3 association. We first sought to map MLK3 association domain/s within WDR62. Towards this end, we co-transfected HEK-293 cells with HAMLK3 and a series of GST-fused WDR62 fragments encompassing the WD40 domain (a.a. 1-733), the middle fragment domain (a.a. 7331018) and the C-terminal domain (a.a. 10181523) (Figure 2A).

Twenty-four hours later, cell lysates were prepared and incubated with glutathione beads. Following extensive washes, GST-containing protein complexes were eluted. Western blot analysis revealed that neither GST alone nor the GST-fused WD40 domain could pull down HA-MLK3. The expression level of WD40 was relatively low, nevertheless, was significantly detectable. This level of expression was sufficient to display interaction with other proteins (data not shown). Importantly, efficient precipitation was observed with GST-fused middle and moderate precipitation with the C-terminal WDR62 fragments (Figure 2B, full gel is shown in Supplemental Figure 1). To further map the association domains within WDR62, we designed a series of plasmids encoding smaller WDR62 fragments fused to the Myc epitope tag (Figure 2C). HEK-293 cells were transfected with HA-tagged MLK3 together with the various Myc-tagged WDR62 fragments for co-IP assays. Western blot analysis revealed two sep- 
arable domains within WDR62 that interact with MLK3. The C-terminal fragment harboring MKK4/7 and JNK docking domains (a.a. 1212-1523) did not associate with HA-MLK3 (Figure 2D, full gel is shown in Supplemental Figure 2).

\section{Mapping MLK3 association domains with WDR62}

MLK proteins are composed of modular structural domains including an $\mathrm{N}$-terminal SH3 domain (a.a.1-114), a kinase domain (a.a.115-399), a leucine zipper and CRIB domain (a.a. 400-591) and a C-terminal domain (a.a. 592-847). To determine which of the MLK3 domains associates with the WDR62 fragments, we created a series of plasmids encoding HA-tagged MLK3 domains and GSTtagged WDR62 fragments for GST pull down assays. Control pull down with GST protein did not precipitate MLK3 fragments (Supplemental Figure 3).

Significantly, two distinct domains within MLK3, namely the kinase domain (a.a. 115399) and C-terminal domain (a.a. 592-847), coimmunoprecipitated with both WDR62 7331018 and 1018-1284 fragments (Figure 3B). Upon longer exposure, the glycine rich domain displayed precipitated with WDR62 733-1018GST fragment but not with WDR62 1018-1284 (Figure 3B). The expression of the MLK3 Nterminal SH3 domain (1-114) was undetectable in the cell lysates, and therefore we cannot rule out the possibility that it associates with both WDR62 fragments.

\section{CDC42QL disrupts WDR62-MLK3 association}

Our finding that association between WDR62 and MLK3 involves the MLK3 kinase domain may suggest that WDR62 keeps MLK3 in an inactive state. To test this, we examined whether WDR62-MLK3 association can be disrupted by activating MLK3 via CDC42. Towards this end, we co-expressed WDR62-GST fragments (Figure 4A) together with full length HA-MLK3 in the absence or presence of the activated form of CDC42 (AU5-CDC42QL) for GST pull down assays.

Western blot analysis of the eluted protein complexes revealed that MLK3 association with the WDR62 fragments was disrupted in the presence of CDC42QL (Figure 4B). To verify CDC42QL activity, MLK3 phosphorylation state was verified by Western blot analysis using anti-phospho-MLK3 antibody (Supplemental Figure 4). Distruption of WDR62-MLK3 association, was not dependent on MLK3 kinase activity, since the presence of CDC42QL also disrupted interaction between kinase-dead MLK3 (MLK3-K144A) and WDR62 fragments (Figure 4C). While the WDR62 fragment corresponding to 733-1018 was partially

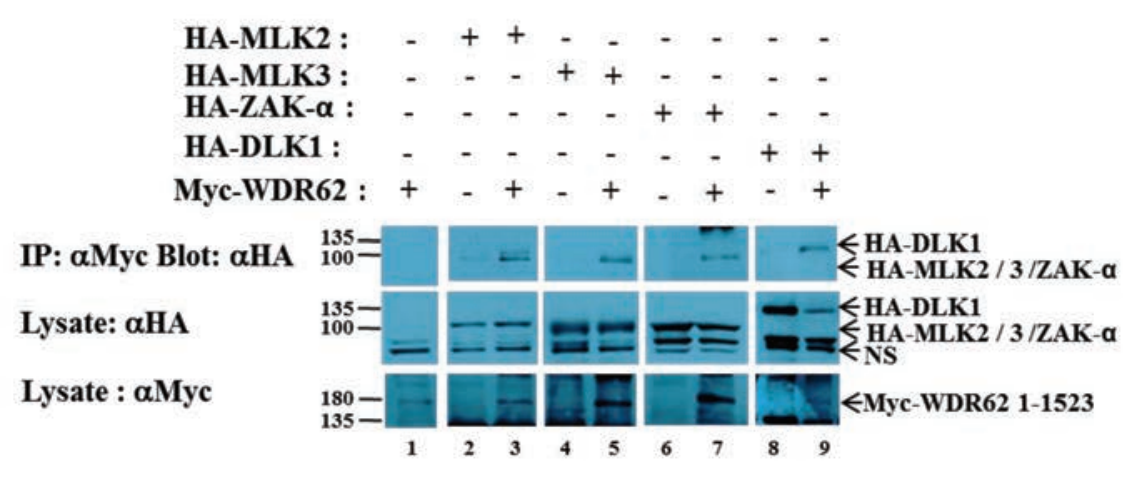

Figure 1. WDR62 associates with all members of the MLK family. HEK-293T cells were transfected with the indicated plasmids. Cell lysates were subjected to immuno-precipitation (IP) with anti-Myc antibodies followed by Western blotting with anti-HA antibodies (upper panel). The expression level of transfected HA-MLK and Myc-WDR62 proteins were determined by blotting the total cell lysate with anti-HA and anti-Myc antibodies, respectively (middle and lower panels). The migration of the relevant proteins is indicated by arrows. Non-specific bands (NS) recognized by anti-HA antibodies are indicated.

(ated.

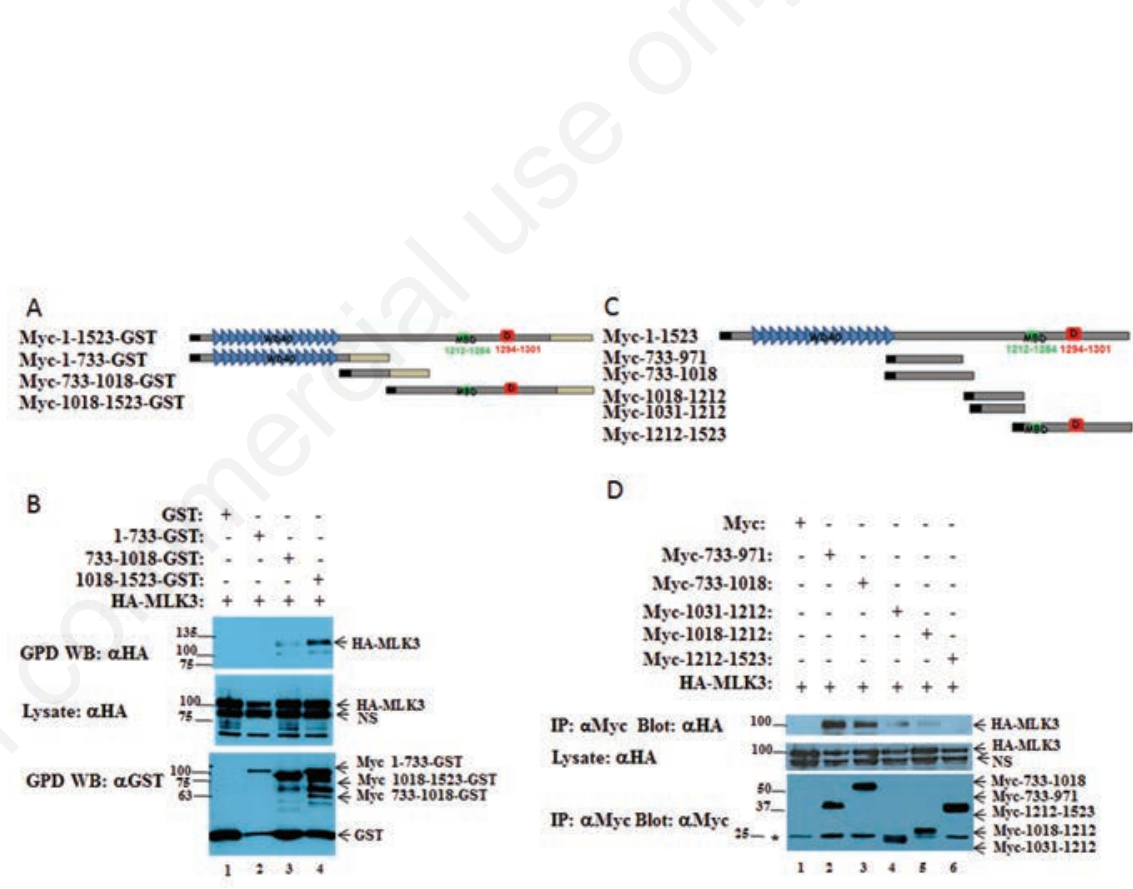

Figure 2. Two WDR62 separable domains associate with HA-MLK3. A) Schematic representation of the WDR62 deletion constructs used in the experiments. The light grey rectangle represents the position of the GST tag. The black square represents the position of the Myc epitope-tag. The WD40 domain (blue, WD40), MKK7 binding domain (Green, MBD), and the JNK docking domain (red, D) are indicated. B) HEK-293T cells were transfected with the indicated plasmids. GST-containing complexes were isolated from cell lysates by GST pull down (GPD). Eluted protein complexes were subjected to Western blotting with either anti-HA (upper panel) or anti-GST (lower panel) antibodies. The expression level of transfected HA-MLK3 was determined by blotting the total cell lysate with an anti-HA antibody (middle panel). The migration of the relevant proteins is indicated by arrows. C) Schematic representation of the WDR62 deletion constructs used in the experiments. The black square represents the position of the Myc epitope-tag. D) HEK-293T cells were transfected with the indicated plasmids. Cell lysates were subjected to immuno-precipitation with anti-Myc antibodies followed by Western blotting with either anti-HA (upper panel) or anti-Myc (lower panel) antibodies. The expression level of transfected HA-MLK3 was determined by blotting the total cell lysate with an anti-HA antibody (middle panel). The migration of the relevant proteins is indicated by arrows. Asterisk $(*)$ represents non-specific precipitation of the IgG light chain at $25 \mathrm{kDa}$. 
disrupted, the association of the WDR62 fragment 1018-1284 with MLK3 could not be observed in the presence of activated CDC42.

\section{JNK activation does not affect WDR62-MLK3 association}

A possible explanation for CDC42 disruption of WDR62-MLK3 association may be through JNK activation. Previously we showed that JNK activation does not affect WDR62-JNK association. ${ }^{21}$ Since CDC42 activates JNK, 16,35 we asked whether JNK activation is responsible for the disruption of WDR62-MLK3 association. We therefore examined WDR62-MLK3 association in the presence of either activatedJNK using the JNKK2-JNK1 chimera or kinasedead JNK using the JNKK2-JNK1KM mutant ${ }^{33}$ (Figure 5).

We verified JNK activation by probing Western blots of cell lysates with anti-phosphoJNK antibodies. Indeed, we observed a strong JNKK2-JNK1 phosphorylation (Figure 5, middle panel, anti-phospho-JNK, lane 2). In addition, endogenous p-JNK was observed in all MLK3 overexpressed lanes. Co-IP experiments revealed that neither active nor inactive JNK affected WDR62-MLK3 association (Figure 5, upper panel).

\section{Discussion}

Signaling networks are organized in a modular structure composed of multiple kinases that are coordinated by scaffold proteins. It is long believed that scaffold proteins are not just bystanders but rather actively participate in signal transmission. ${ }^{13}$ WDR62 is a bona fide JNK scaffold protein that was shown to associate with MAPK and MAP2K of the JNK tier. ${ }^{22}$ Here we show that WDR62 independently associates with MLK3, an additional kinase in the JNK tier. MLK3 is part of the MLK family of MAP3Ks. In un-stimulated cells, the association of each protein in the JNK tier with WDR62 is independent. Moreover, the docking domain of each kinase within the JNK tier is distributed along WDR62 in a sequential manner. Each of the kinases (JNK-MKK4/7-MLK3) possesses a unique docking domain within WDR62 with no apparent overlap. While in JIP the JNK-MKK4/7-MLK tier is organized from Nto-C terminal order, in WDR62 the tier is organized in the reverse orientation, namely, MLK3-MKK4/7-JNK, from N-to-C terminal. In this study we identified two domains within WDR62 that associate with two domains of MLK3. The association between WDR62 fragments 733-1018 and 1018-1212 with MLK3 seems to be less efficient (Figures 2D and 4B). However, we do observe the opposite association in other experiments (Figures 2B and

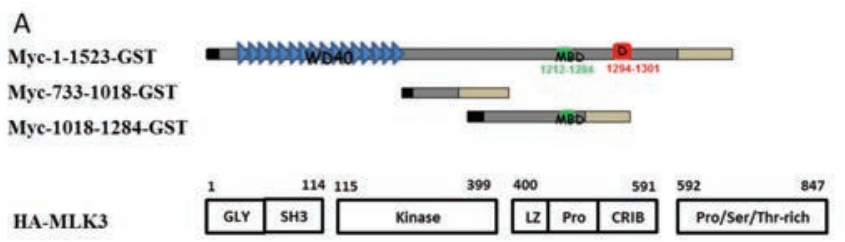

B

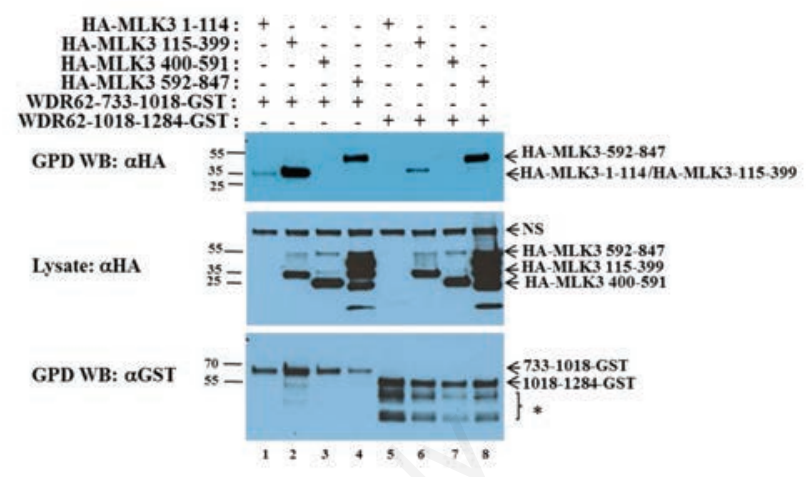

Figure 3. MLK3 kinase and C-terminal domains associate with the two WDR62-MLK3 binding domains. A) Schematic representation of the WDR62 and MLK3 constructs used in the experiments. The light grey rectangle represents the position of the GST-tag. MLK3 fragments were $\mathrm{N}$-terminaly HA-tagged. B) HEK-293T cells were transfected with the indicated plasmids. GST-containing complexes were isolated from cell lysates by GST pull down (GPD). Eluted protein complexes were subjected to Western blotting with either anti-HA (upper panel) or anti-GST (lower panel) antibodies. The expression level of transfected HA-MLK3 was determined by blotting the total cell lysate with an antiHA antibody (middle panel). The migration of the relevant proteins is indicated by arrows. Degradation products are indicated by asterisk*.

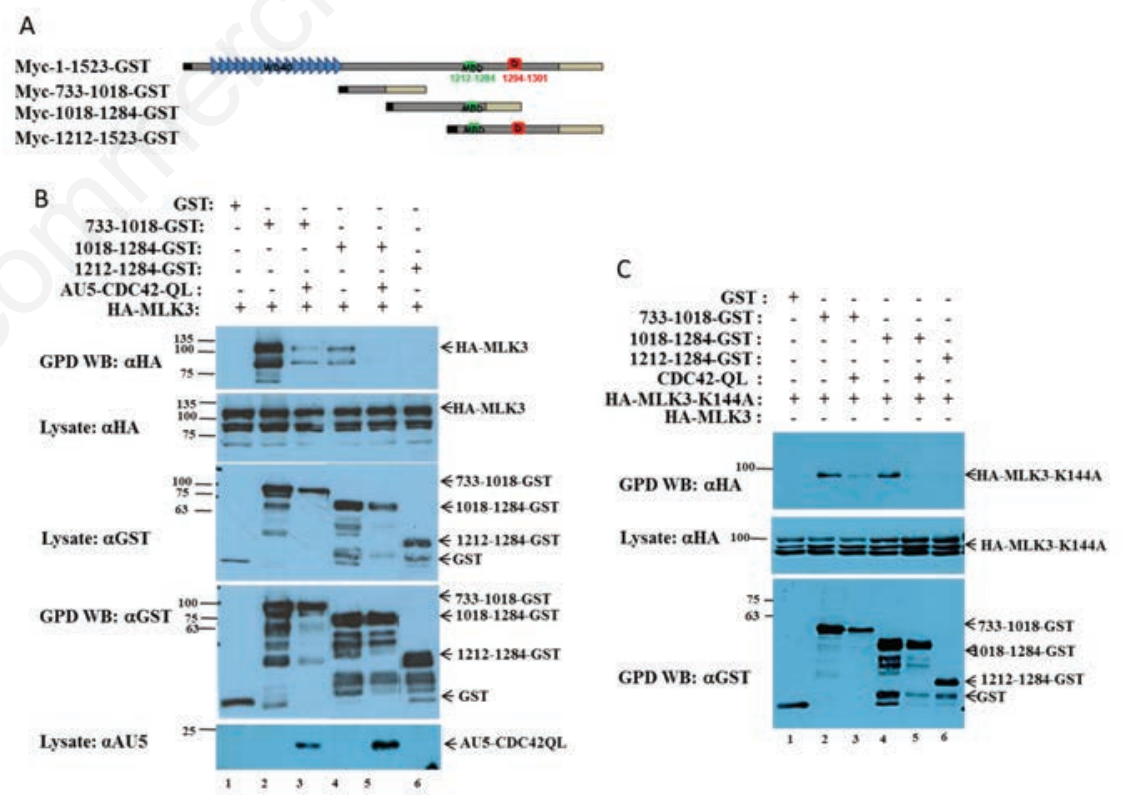

Figure 4. CDC42 disrupts WDR62-MLK3 association. A) Schematic representation of the WDR62 deletion constructs used in the experiments. The light grey rectangle represents the position of the GST tag. B) HEK-293T cells were transfected with the indicated GST-WDR62 and HA-MLK3 plasmids in the presence or absence of a plasmid encoding activated CDC42 (CDC42 QL). GST-containing complexes were isolated from cell lysates by GST pull down (GPD). Eluted protein complexes were subjected to Western blotting with either anti-HA (upper panel), anti-GST and anti-AU5 antibodies (lower panel). The expression level of transfected HA-MLK3 and GST-WDR62 fragments was determined by blotting the total cell lysate with an anti-HA and anti-GST antibodies, respectively (middle panels). The migration of the relevant proteins is indicated by arrows. C) As described in B except that a plasmid encoding kinase inactive HA-MLK3 (HA-MLK3-K144M) was used in all the transfections. 
4C). This is mainly due to the fact that coimmuno-precipitation are mainly qualitative assay and not quantitative. Interestingly, the domains do not share any sequence or structural homology yet, these fragments are able to form pairwise associations. The structural determinants that permit such binding flexibility are yet to be explored. One of the domains within MLK3 that associates with WDR62 includes the MLK3 kinase domain. Similarly, DLK1 kinase domain was shown to associate with JIP1 JNK scaffold protein.32 Analysis of interactions between WDR62 and other MLK family members revealed that DLK1 associates with the N-terminal WD40 domain (1-733) and WDR62 733-1018 but not with WDR62 1018-1284 domain (Hadad and Aronheim unpublished results). Importantly, the DLK1 kinase domain (a.a. 1-407) was found to interact with WDR62 (Hadad and Aronheim unpublished results). Interestingly, two domains within DLK1 corresponding to a.a. 1-179 and a.a. 342-407 were shown to interact with JIP C-terminal domain. 32 The JIPDLK1 interaction under basal conditions suggests that DLK1 is held in a monomeric unphosphorylated and catalytically inactive state. ${ }^{36}$ MLK3 is known to be auto-inhibited through the internal association of the N-terminal SH3 domain with MLK3 CRIB domain. ${ }^{37}$ This led us to hypothesize that WDR62-MLK3 association maintains MLK3 in a monomeric inactive form. Indeed, we observe that MLK3 auto-phosphorylation in the activation loop is inhibited in the presence of WDR62. Activation of the MLK3-MKK4/7-JNK pathway by CDC42 is sufficient to disrupt WDR62-MLK3 association. However, we show that both MLK3 and JNK kinase activity are dispensable for the disruption of the WDR62-MLK3 complex. This suggests that following CDC42 activation, CDC42 directly competes with WDR62 for MLK3 binding. Thus, MLK3 dissociation may allow its dimerization and activation following tier activation.

The MLK3 leucine zipper domain forms inefficient dimers with DLK protein.16 Therefore, the finding that both common and unique docking domains for DLK1 and MLK3 are found within the WDR62 scaffold protein may suggest that WDR62 serves as a dimerization interface platform for multiple members of the MLK family to coordinate JNK activation. In conclusion, we have characterized the association between the JNK scaffold protein, WDR62, and MLK3. We mapped two unique MLK3 association domains within WDR62 and two WDR62 association domains within MLK3. WDR62-MLK3 association occurs in a JNK and MKK independent manner. The WDR62-MLK3 complex dissociates following activation by the upstream small GTPase, CDC42 but not following JNK activation.

\section{References}

1. Shaul YD, Seger R. The MEK/ERK cascade: from signaling specificity to diverse functions. Biochim Biophys Acta 2007;1773: 1213-26.

2. Engelberg D, Perlman R, Levitzki A. Transmembrane signaling in Saccharomyces cerevisiae as a model for signaling in metazoans: state of the art after 25 years. Cell Signal 2014;26:2865-78.

3. Bogoyevitch MA, Kobe B. Uses for JNK: the many and varied substrates of the c-Jun Nterminal kinases Microbiol Mol Biol R 2006;70:1061-95.

4. Bogoyevitch MA, Ngoei KR, Zhao TT, et al. c-Jun N-terminal kinase (JNK) signaling: recent advances and challenges. Biochim Biophys Acta 2010;1804:463-75.

5. Johnson GL, Nakamura K. The c-jun kinase/stress-activated pathway: regulation, function and role in human disease. Biochim Biophys Acta 2007;1773:1341-8.

6. Weston CR, Davis RJ. The JNK signal transduction pathway. Current Opin Cell Biol 2007;19:142-9.

7. Enslen H, Davis RJ. Regulation of MAP kinases by docking domains. Biol Cell 2001;93:5-14.

8. Bardwell L. Mechanisms of MAPK signalling specificity. Biochem Soc T 2006; 34:837-41.

9. Bardwell AJ, Frankson E, Bardwell L. Selectivity of docking sites in MAPK kinases. J Biol Chem 2009;284:13165-73.

10. Weston CR, Lambright DG, Davis RJ. MAP kinase signaling specificity. Science 2002;296:2345-7.

11. Dhanasekaran DN, Kashef K, Lee CM, et al. Scaffold proteins of MAP-kinase modules. Oncogene 2007;26:3185-202.

12. van Drogen F, Peter M. MAP kinase cascades: scaffolding signal specificity. Curr Biol 2002;12:R53-5.

13. Smith FD, Scott JD. Scaffolding proteins: not such innocent bystanders. Curr Biol 2013;23:R515-7.

Lysate: $\alpha$ Flag

Lysate: $\alpha$-Myc

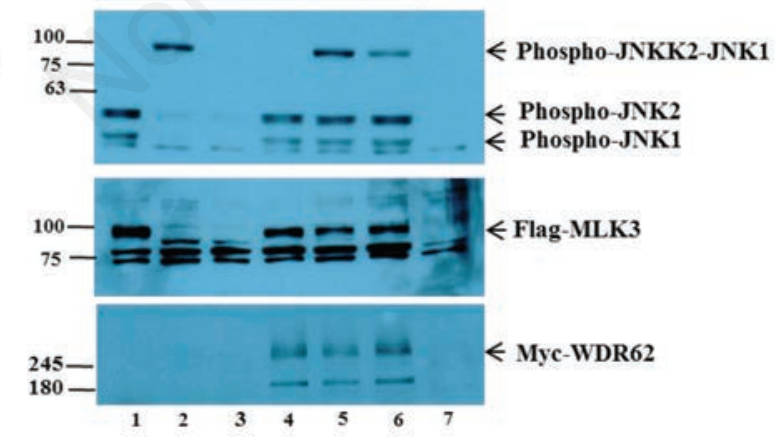

14. Sehgal V, Ram PT. Network Motifs in JNK Signaling. Genes Cancer 2013;4:409-13.

15. Haeusgen W, Herdegen T, Waetzig V. The bottleneck of JNK signaling: molecular and functional characteristics of MKK4 and MKK7. Eur J Cell Biol 2011;90:536-44.

16. Gallo KA, Johnson GL. Mixed-lineage kinase control of JNK and p38 MAPK pathways. Nat Rev Mol Cell Biol 2002;3:663-72.

Figure 5. JNK activation does not disrupt WDR62-MLK3 association A. HEK-293T cells were transfected with plasmids encoding Myc-WDR62, Flag-MLK3, activated JNK chimera (HA-JNKK2-JNK1), and kinase-dead JNK (HA-JNKK2-JNK1-KM) as indicated. Co-IP containing complexes were isolated from cell lysates with anti-Myc antibodies. Eluted protein complexes were subjected to Western blotting with anti-Flag antibodies (upper panel). The expression level of transfected Flag-MLK3 and Myc-WDR62 was determined by blotting the total cell lysate with anti-Flag and anti-Myc antibodies, respectively (lower panels). JNK activation was verified by blotting the total cell lysate with anti-phospho-JNK antibodies (middle panel). The migration of the relevant proteins is indicated by arrows.

17. Whitmarsh AJ. The JIP family of MAPK scaffold proteins. Biochem Soc T 2006;34:828-32.

18. Holmberg C, Katz S, Lerdrup M, et al. A novel specific role for I kappa B kinase complex-associated protein in cytosolic stress signaling. J Biol Chem 2002;277: 
31918-28.

19. Kim GH, Park E, Kong YY, Han JK. Novel function of POSH, a JNK scaffold, as an E3 ubiquitin ligase for the Hrs stability on early endosomes. Cell Signal 2006;18:55363.

20. Broder YC, Katz S, Aronheim A. The Ras recruitment system, a novel approach to the study of protein-protein interactions. Curr Biol 1998;8:1121-4.

21. Wasserman T, Katsenelson K, Daniliuc S, et al. novel c-Jun $\mathrm{N}$-terminal kinase (JNK)-binding protein WDR62 is recruited to stress granules and mediates a nonclassical JNK activation. Mol Biol Cell 2010;21:117-30.

22. Cohen-Katsenelson K, Wasserman T, Khateb S, et al. Docking interactions of the JNK scaffold protein WDR62. Biochem J 2011;439:381-90.

23. Bogoyevitch MA, Yeap YY, Qu Z, et al. WD40-repeat protein 62 is a JNK-phosphorylated spindle pole protein required for spindle maintenance and timely mitotic progression. J Cell Sci 2012;125:5096-109.

24. Bilguvar K, Ozturk AK, Louvi A, et al. Whole-exome sequencing identifies recessive WDR62 mutations in severe brain malformations. Nature 2010;467:207-10.

25. Nicholas AK, Khurshid M, Desir J, et al.
WDR62 is associated with the spindle pole and is mutated in human microcephaly. Nat Genet 2010;42:1010-4.

26. Yu TW, Mochida GH, Tischfield DJ, et al. Mutations in WDR62, encoding a centrosome-associated protein, cause microcephaly with simplified gyri and abnormal cortical architecture. Nat Genet 2010;42: 1015-20.

27. Xu D, Zhang F, Wang Y, et al. Microcephalyassociated protein WDR62 regulates neurogenesis through JNK1 in the developing neocortex. Cell Rep 2014;6:104-16.

28. Cohen-Katsenelson K, Wasserman T, Darlyuk-Saadon I, et al. Identification and analysis of a novel dimerization domain shared by various members of JNK scaffold proteins. J Biol Chem 2013; 288:7294304.

29. Lim NR, Yeap YY, Zhao TT, et al. Opposing roles for JNK and Aurora A in regulating WD40-Repeat Protein 62 association with spindle microtubules. J Cell Sci 2014;128:527-40.

30. Stanhill A, Haynes CM, Zhang Y, et al. An arsenite-inducible $19 \mathrm{~S}$ regulatory particleassociated protein adapts proteasomes to proteotoxicity. Mol Cell 2006;23:875-85.

31. Korkina 0, Dong Z, Marullo A, et al. The MLK-related kinase (MRK) is a novel
RhoC effector that mediates lysophosphatidic acid (LPA)-stimulated tumor cell invasion. J Biol Chem 2013;288:5364-73.

32. Mooney LM, Whitmarsh AJ. Docking interactions in the c-Jun N-terminal kinase pathway. J Biol Chem 2004;279:11843-52.

33. Zheng $\mathrm{C}$, Xiang J, Hunter T, Lin A. The JNKK2-JNK1 fusion protein acts as a constitutively active c-Jun kinase that stimulates c-Jun transcription activity. J Biol Chem 1999;274:28966-71.

34. Batard P, Jordan M, Wurm F. Transfer of high copy number plasmid into mammalian cells by calcium phosphate transfection. Gene 2001;270:61-8.

35. Coso OA, Chiariello M, Yu JC, et al. The small GTP-binding proteins Racl and Cdc42 regulate the activity of the JNK/SAPK signaling pathway. Cell 1995;81: 1137-46.

36. Nihalani D, Meyer D, Pajni S, Holzman LB. Mixed lineage kinase-dependent JNK activation is governed by interactions of scaffold protein JIP with MAPK module components. EMBO J 2001;20:3447-58.

37. Zhang H, Gallo KA. Autoinhibition of mixed lineage kinase 3 through its Src homology 3 domain. J Biol Chem 2001;276: 45598-603. 